\title{
EQUICONVERGENCE OF MATRIX TRANSFORMATIONS
}

\author{
K. A. JUKES
}

ABSTRACT. Equiconvergence of matrix transformations is related to the existence of Tauberian constants. Agnew's result on the equiconvergence of Cesàro and Riesz means is shown to be best possible. Finally, equiconvergence of equivalent arithmetical summation methods related to the prime number theorem is investigated.

1. Introduction. Agnew [1, Theorems 3.1, 4.1] showed directly that, for $r>0$, the Cesàro and Riesz transforms

$$
\begin{aligned}
& C_{r}(n)=\left(\begin{array}{c}
n+r \\
r
\end{array}\right)^{-1} \sum_{k=0}^{n}\left(\begin{array}{c}
n-k+r \\
n-k
\end{array}\right) a_{k}, \\
& R_{r}(n)=\sum_{k=0}^{n}(1-k / n)^{r} a_{k},
\end{aligned}
$$

of a given series $\sum a_{k}$ are equiconvergent, i.e. $C_{r}(n), R_{r}(n)$ exist for each $n$ and $\lim _{n \rightarrow \infty}\left\{C_{r}(n)-R_{r}(n)\right\}=0$, whenever either $a_{n} \rightarrow 0$, or $\sum_{k=0}^{n} k a_{k}=O(n+$ $1)$, as $n \rightarrow \infty$. It is well known that the two transforms are equivalent in the sense that the convergence of either implies that of the other to the same limit. What is of interest is that, whether or not either transform converges, the transforms are equiconvergent for a wide class of series, a point which does not follow from classic facts about the Cesàro and Riesz transforms. Agnew's results exemplify two means of deducing equiconvergence from results on the existence of Tauberian constants.

Write

$$
B_{n}(a)=\sum b_{n k} a_{k}, \quad C_{n}(a)=\sum c_{n k} a_{k}, \quad \Delta\left(a_{n k}\right)=a_{n k}-a_{n, k+1},
$$

where $\left(a_{n k}\right),\left(b_{n k}\right),\left(c_{n k}\right)$ are three given matrices, and suppose $(g(n))$ is a complex sequence satisfying $g(n) \neq 0$ for each $n .^{1}$

Maddox [7] (see also Jukes [3, p. 748]) proved

THEOREM M1. $B_{n}(a), C_{n}(a)$ exist for each $n$ and there is a constant $M_{1}$ such that

$$
\lim _{n} \sup \left|B_{n}(a)-C_{n}(a)\right| \leqslant M_{1} A_{1}
$$

whenever

Received by the editors May 8, 1974.

AMS (MOS) subject classifications (1970). Primary 40D10; Secondary 10H25.

${ }^{1}$ Unless otherwise stated all sums run from $k=0$ to $k=\infty$. 


$$
A_{1}=\lim \sup _{n}\left|g(n) a_{n}\right|<\infty
$$

if and only if

(2) $b_{n k}-c_{n k} \rightarrow 0$ as $n \rightarrow \infty$ for each $k$,

(3) $\Sigma\left(\left|b_{n k}\right|+\left|c_{n k}\right|\right) /|g(k)|<\infty$ for each $n$, and

$$
D=\limsup _{n} \sum\left|\frac{b_{n k}-c_{n k}}{g(k)}\right|<\infty .
$$

Further, when (2)-(4) hold we may take $M_{1}=D$, and this is then best possible.

TheOREM M2. $B_{n}(a), C_{n}(a)$ exist for each $n$ and there is a constant $M_{2}$ such that

$$
\limsup _{n}\left|B_{n}(a)-C_{n}(a)\right| \leqslant M_{2} A_{2}
$$

whenever

$$
A_{2}=\lim \sup _{n}\left|\sum_{k=0}^{n} \frac{k a_{k}}{(n+1)}\right|<\infty
$$

if and only if

(6) $\left|b_{n k}\right|+\left|c_{n k}\right| \rightarrow 0$ as $k \rightarrow \infty$ for each $n$,

(7) $b_{n k}-c_{n k} \rightarrow 0$ as $n \rightarrow \infty$ for each $k$,

(8) $\sum k\left(\left|\Delta\left(b_{n k} / k\right)\right|+\left|\Delta\left(c_{n k} / k\right)\right|\right)<\infty$ for each $n$, and

$$
D=\lim _{n} \sup \sum k\left|\Delta\left(\frac{b_{n k}-c_{n k}}{k}\right)\right|<\infty .
$$

When (6)-(9) hold, $M_{2}=D$ is best possible.

Trivially the transforms $B_{n}(a), C_{n}(a)$ are equiconvergent if both exist when $A_{i}<\infty$ and either $M_{i}=0$ or $M_{i}$ exists and $A_{i}=0$.

In fact the existence of $B_{n}(a), C_{n}(a)$ together with $M_{2}=0$ is clearly both necessary and sufficient for equiconvergence whenever $A_{2}<\infty$. Agnew's second result supplies an example of this feature. More surprisingly, we have

THEOREM 1. Equiconvergence whenever $A_{1}=0$ is equivalent to the existence of Tauberian constants whenever $A_{1}<\infty$.

Proof. Conditions (2) and (4) are necessary and sufficient for the matrix $\left(\left(b_{n k}-c_{n k}\right) / g(k)\right)$ to map $c_{0}$ into $c_{0}$, while $(3)$ is necessary and sufficient for the existence of $B_{n}(a)$ and $C_{n}(a)$ whenever $\left(a_{k} g(k)\right) \in c_{0}$. Thus equiconvergence whenever $A_{1}=0$ occurs if and only if (2)-(4) hold.

Using Theorem 1 we can obtain Agnew's first result and show that it in a sense best possible. We shall also investigate equiconvergence of certain arithmetical summation methods connected with the prime number theorem. In particular, we examine Ingham's method and the method $\left(D, \mu^{2}(n) / n\right)$, the equivalence of which, up to a factor of $6 / \pi^{2}$, was shown in $[4, p .702]$. 


\section{Cesàro and Riesz means.}

TheOREM 2. Let $r>0$. The Cesàro and Riesz transforms (1) are equiconvergent whenever $n^{\alpha} a_{n} \rightarrow 0$ if and only if $\alpha \geqslant 0$.

Our proof makes use, for real $z$, of the following formula for the quotient of two gamma functions:

(10) $\frac{\Gamma(z+a)}{\Gamma(z+b)}=z^{a-b}\left\{1+\frac{(a-b)(a+b-1)}{2 z}+\frac{C}{z^{2}}\right\}\left(1+O\left(z^{-3}\right)\right)$,

where $|\arg z| \leqslant \pi-\varepsilon(\varepsilon>0)$ and $C=C(a, b)$ is a constant (see, e.g. Luke [6, p. 33, equation (11)]). With $z=n, a=r+1, b=1$ this gives

$$
\begin{aligned}
\frac{\Gamma(n+r+1)}{\Gamma(n+1)} & =n^{r}\left\{1+\frac{r(r+1)}{2 n}+\frac{C}{n^{2}}\right\}\left(1+O\left(n^{-3}\right)\right) \\
& =n^{r}\left\{1+\frac{r(r+1)}{2 n}+\frac{C}{n^{2}}+\frac{D_{n}}{n^{3}}\right\},
\end{aligned}
$$

where $D_{n}=O(1)$. In particular, there exist bounded sequences $\left(A_{k}\right),\left(B_{k}\right)$ such that

$$
\frac{\Gamma(n+r+1)}{\Gamma(n+1)}=n^{r}\left(1+\frac{A_{n}}{n}\right)=n^{r}\left(1+\frac{B_{n}}{n}\right)^{-1} \quad(n=1,2, \ldots),
$$

as stated in [1, (3.11)].

Proof of Theorem 2. Write

$$
\begin{aligned}
& b_{n k}= \begin{cases}\frac{\Gamma(r+n-k+1)}{\Gamma(n-k+1)} \frac{\Gamma(n+1)}{\Gamma(r+n+1)} & (0 \leqslant k \leqslant n), \\
0 & (k>n),\end{cases} \\
& c_{n k}= \begin{cases}(1-k / n)^{r} & (0 \leqslant k \leqslant n), \\
0 & (k>n) .\end{cases}
\end{aligned}
$$

Then for $k<n$,

$$
b_{n k}=\left(1-\frac{k}{n}\right)^{r}\left(1+\frac{A_{n-k}}{n-k}\right)\left(1+\frac{B_{n}}{n}\right)
$$

so (2) is satisfied. Taking $g(0)=1, g(k)=k^{\alpha}(k \geqslant 1)$, (3) is trivially satisfied. If $\alpha \geqslant 0$ then

$$
\begin{aligned}
\sum_{k=0}^{\infty}\left|\frac{b_{n k}-c_{n k}}{g(k)}\right| \leqslant & \sum_{k=1}^{n-1}\left|\left(1-\frac{k}{n}\right)^{r}\left(\frac{A_{n-k}}{n-k}+\frac{B_{n}}{n}+\frac{A_{n-k} B_{n}}{(n-k) n}\right)\right| \\
& +\frac{\Gamma(r+1) \Gamma(n+1)}{n^{\alpha} \Gamma(r+n+1)} \\
= & O\left(\frac{1}{n^{r}} \sum_{k=1}^{n-1}(n-k)^{r-1}\right)+o(1) \\
= & O(1)
\end{aligned}
$$


as $n \rightarrow \infty$, whence equiconvergence follows. If $\alpha<0$ then by (11) and (12), for $n$ sufficiently large,

$$
\begin{aligned}
& \sum_{k=0}^{\infty}\left|\frac{b_{n k}-c_{n k}}{g(k)}\right| \\
& \geqslant \sum_{1<k<n / 2} k^{-\alpha}\left|\frac{\frac{\Gamma(r+n-k+1)}{\Gamma(n-k+1)}-\frac{\Gamma(r+n+1)}{\Gamma(n+1)}\left(1-\frac{k}{n}\right)^{r}}{n^{r}\left(1+A_{n} / n\right)}\right| \\
& \geqslant \frac{1}{2 n^{r}} \sum_{1<k<n / 2} k^{-\alpha}(n-k)^{r} \\
& \cdot \mid\left(1+\frac{r(r+1)}{2(n-k)}+\frac{C}{(n-k)^{2}}+\frac{D_{n-k}}{(n-k)^{3}}\right) \\
& -\left(1+\frac{r(r+1)}{2 n}+\frac{C}{n^{2}}+\frac{D_{n}}{n^{3}}\right) \\
& =\frac{1}{2 n^{r+1}} \sum_{1<k<n / 2} k^{1-\alpha}(n-k)^{r-1} \\
& \cdot\left|\frac{r(r+1)}{2}+C\left(\frac{2}{n-k}-\frac{k}{n(n-k)}\right)+\frac{n D_{n-k}}{(n-k)^{2} k}-\frac{(n-k) D_{n}}{n^{2} k}\right| \\
& \geqslant \frac{1}{2 n^{r+1}} \sum_{1<k<n / 2} k^{1-\alpha}(n-k)^{r-1} \frac{r(r+1)}{4}
\end{aligned}
$$

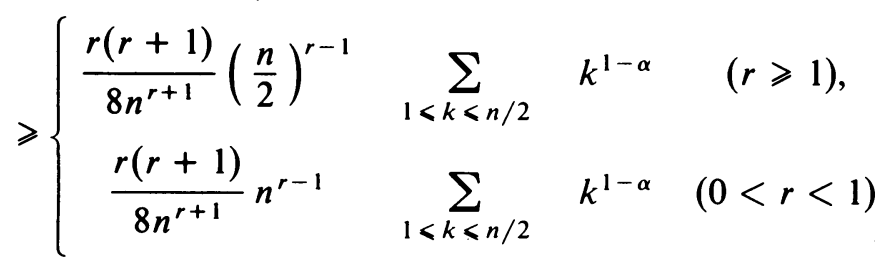

$$
\begin{aligned}
& \rightarrow \infty \text { as } n \rightarrow \infty \text {, }
\end{aligned}
$$

completing the proof.

Agnew's proof of (12) would seem to require a stronger form of Stirling's formula than the usual $n !=\sqrt{2 \pi} n^{n+1 / 2} e^{-n} e^{\theta(n)}$, where $\theta(n)=o(1)$. For positive integers $r$, both this approach and use of (10) can be avoided by the following simple argument:

$$
\begin{aligned}
0 & \leqslant\left(\frac{(n+r) !}{n ! n^{r}}-1\right) n=\frac{(n+1) \ldots(n+r)-n^{r}}{n^{r-1}} \\
& \leqslant \frac{(n+r)^{r}-n^{r}}{n^{r-1}}=\frac{r x^{r-1}}{n^{r-1}} \quad(n<x<n+r) \\
& \leqslant \frac{r(n+r)^{r-1}}{n^{r-1}}=O(1)
\end{aligned}
$$


as $n \rightarrow \infty$. A similar use of the mean value theorem also simplifies the required estimate in the case $\alpha<0$ when $r$ is a positive integer.

3. Arithmetical summation methods. Let $h(n)$ denote a real-valued function on the positive integers with $h(1)=1$. A series $\Sigma_{1}^{\infty} a_{n}$ is called $(D, h(n))$-summable to the sum $A$ if

$$
\lim _{n \rightarrow \infty} \frac{1}{n} \sum_{k=1}^{n} k a_{k} \sum_{r<n / k} r h(r)=A .
$$

Ingham's method (I) is given by $h(n)=1 / n$. Consider the $\left(D, \mu^{2}(n) / n\right)$ and $6(\mathrm{I}) / \pi^{2}$ transforms given by

$$
b_{n k}=\frac{k}{n} \sum_{r<n / k} \mu^{2}(r), \quad c_{n k}=\frac{6}{\pi^{2}} \frac{k}{n}\left[\frac{n}{k}\right],
$$

respectively, where $[x]$ denotes the integer part of $x$.

TheOREM 3. The $\left(D, \mu^{2}(n) / n\right)$ and $6(\mathrm{I}) / \pi^{2}$ transforms are equiconvergent whenever $n^{\alpha} a_{n} \rightarrow 0$ if and only if $\alpha \geqslant 1$.

Proof. Taking $g(k)=k^{\alpha}$, (3) is trivially satisfied for any $\alpha$, while (2) follows from the well-known estimate

$$
Q(x) \equiv \sum_{r<x} \mu^{2}(r)=6 x / \pi^{2}+O(\sqrt{ })
$$

for the square-free numbers (see, e.g., Hardy and Wright [2, Theorem 333]). Further,

$$
\begin{aligned}
S & \equiv \sum_{k=1}^{n}\left|\frac{k n^{-1} \sum_{r \leqslant n / k} \mu^{2}(r)-6 \pi^{-2} k n^{-1}\left[n k^{-1}\right]}{k^{\alpha}}\right| \\
& =O\left(\frac{1}{\sqrt{ } n} \sum_{k=1}^{n} k^{1 / 2-\alpha}\right) .
\end{aligned}
$$

If $\alpha \geqslant 1$ then $S=O(1)$, while for $\alpha<1$,

$$
S \geqslant \sum_{n / 2<k<n} \frac{k^{1-\alpha}}{n}\left(1-\frac{6}{\pi^{2}}\right) \rightarrow \infty \quad \text { as } n \rightarrow \infty .
$$

For these transforms, if $\alpha \geqslant 1$ the Tauberian constant $M_{1}$, in fact, exists as a limit.

THEOREM 4. Let $g(k)=k^{\alpha}, \alpha \geqslant 1$. Then, for the $\left(D, \mu^{2}(n) / n\right)$ and $6(\mathrm{I}) / \pi^{2}$ transforms,

$$
M_{1}= \begin{cases}0 & (\alpha>1), \\ \int_{0}^{1}\left|Q\left(\frac{1}{x}\right)-\frac{6}{\pi^{2}}\left[\frac{1}{x}\right]\right| d x & (\alpha=1)\end{cases}
$$

(the convergence of the integral following from (14)).

Proof. If $\alpha>1, M_{1}=0$ follows from (15). If $\alpha=1$ we have the Riemann 
sum

$$
S=\sum_{k=1}^{n} \frac{1}{n}\left|Q\left(\frac{n}{k}\right)-\frac{6}{\pi^{2}}\left[\frac{n}{k}\right]\right|,
$$

whence the result.

A similar calculation follows if we compare the $\left(D, \mu^{2}(n) / n\right)$ transform with the factored partial sum given by

$$
c_{n k}= \begin{cases}6 / \pi^{2} & (1 \leqslant k \leqslant n), \\ 0 & (k>n),\end{cases}
$$

the resulting Tauberian constant $M_{1}$ being

$$
M_{1}= \begin{cases}0 & (\alpha>1), \\ \int_{0}^{1}\left|Q\left(\frac{1}{x}\right)-\frac{6}{\pi^{2} x}\right| d x & (\alpha=1) .\end{cases}
$$

In the case of (I) and partial sums we showed [3, Theorem 4] that $M_{1}=1-\gamma$, where $\gamma$ is Euler's constant. The integral

$$
\int_{0}^{1} Q\left(\frac{1}{x}\right)-\frac{6}{\pi^{2} x} d x
$$

can be explicitly evaluated. However, Stark [8] showed that $Q(1 / x)-6 / \pi^{2} x$ changes sign infinitely often as $x \rightarrow 0+$. Though this fact prevents direct explicit evaluation of $M_{1}$ above, the evaluation of (16) is of some interest. The following version, shorter than my own, was kindly suggested by the referee:

Write $M(x)=\Sigma_{n<x} \mu(n)$. Now

$$
\int_{0}^{1} Q\left(\frac{1}{x}\right)-\frac{6}{\pi^{2} x} d x=\int_{1}^{\infty} \frac{Q(t)}{t^{2}}-\frac{6}{\pi^{2} t} d t .
$$

But

$$
Q(t)=\sum_{d<\vee t} \mu(d)\left[\frac{t}{d^{2}}\right]
$$

(see the proof of [2, Theorem 333]), whence

$$
\begin{aligned}
\int_{1}^{n} \frac{Q(t)}{t^{2}} d t & =\int_{1}^{n} \frac{1}{t^{2}} \sum_{d<\vee t} \mu(d)\left[\frac{t}{d^{2}}\right] d t \\
& =\sum_{d<\vee n} \mu(d) \int_{d^{2}}^{n} \frac{1}{t^{2}}\left[\frac{t}{d^{2}}\right] d t \\
& =\sum_{d<\vee n} \frac{\mu(d)}{d^{2}} \int_{1}^{n / d^{2}} \frac{[u]}{u^{2}} d u \\
& =\sum_{d<\vee n} \frac{\mu(d)}{d^{2}}\left(\sum_{k<n / d^{2}} \frac{1}{k}-\left[\frac{n}{d^{2}}\right] \frac{d^{2}}{n}\right)
\end{aligned}
$$

Since 


$$
\sum_{k<x} \frac{1}{k}=\log x+\gamma+O\left(\frac{1}{x}\right)
$$

and

$$
\sum_{d<\vee n} \frac{\mu(d)}{d^{2}}=\frac{6}{\pi^{2}}+O\left(\frac{1}{\sqrt{ } n}\right)
$$

then

$$
\begin{aligned}
\int_{1}^{\infty} \frac{Q(t)}{t^{2}}-\frac{6}{\pi^{2} t} d t & =\lim _{n \rightarrow \infty}\left(\int_{1}^{n} \frac{Q(t)}{t^{2}} d t-\frac{6}{\pi^{2}} \log n\right) \\
& =\lim _{n \rightarrow \infty}\left\{\sum_{d<\vee n} \frac{\mu(d)}{d^{2}}(\gamma-2 \log d)\right. \\
& \left.-\frac{1}{n} \sum_{d<\vee n} \mu(d)\left[\frac{n}{d^{2}}\right]+o(1)\right\} \\
& =\frac{6 \gamma}{\pi^{2}}-2 \sum_{d=1}^{\infty} \frac{\mu(d) \log d}{d^{2}}-\lim _{n \rightarrow \infty} \frac{1}{n} Q(n) \\
& =\frac{6}{\pi^{2}}(\gamma-1)-2 \sum_{d=1}^{\infty} \frac{\mu(d) \log d}{d} .
\end{aligned}
$$

Thus, by $[2, \S 17.7]$,

$$
\int_{0}^{1} Q\left(\frac{1}{x}\right)-\frac{6}{\pi^{2} x} d x=\frac{6}{\pi^{2}}(\gamma-1)-\frac{72}{\pi^{4}} \sum_{d=1}^{\infty} \frac{\log d}{d^{2}}=0.4359 \ldots
$$

Concerning equiconvergence whenever $A_{2}<\infty$, first note

THEOREM 5. Tauberian constants $M_{2}$ do not exist for comparisons of conservative matrices with nonconservative matrices.

Proof. Suppose (5) holds whenever $A_{2}<\infty$, where $\left(b_{n k}\right)$ is a conservative matrix. Now $\Sigma a_{k}$ convergent $\Rightarrow k a_{k} \rightarrow 0(C, 1) \Rightarrow A_{2}=0 \Rightarrow B_{n}(a)-C_{n}(a)$ $\rightarrow 0$. But $\sum a_{k}$ convergent $\Rightarrow \lim _{n} B_{n}(a)$ exists and so $\left(c_{n k}\right)$ is conservative.

Since (I) and $\left(D, \mu^{2}(n) / n\right)$ are not conservative, then for comparisons with conservative methods, equiconvergence whenever $A_{2}<\infty$ is false. Further, in contrast to Agnew's result for Cesàro and Riesz means,

THEOREM 6. The $\left(D, \mu^{2}(n) / n\right)$ and $6(\mathrm{I}) / \pi^{2}$ transforms are not equiconvergent whenever $A_{2}<\infty$.

Proof. With $\left(b_{n k}\right),\left(c_{n k}\right)$ as in (13), (6)-(8) are satisfied. However,

$$
\begin{aligned}
\sum_{k=1}^{\infty} k\left|\Delta\left(\frac{b_{n k}-c_{n k}}{k}\right)\right| & =\left.\sum_{k=1}^{n} \frac{k}{n}\right|_{n /(k+1)<r<n / k}\left(\mu^{2}(r)-6 / \pi^{2}\right) \mid \\
& \geqslant \frac{1}{n} \sum_{\vee n<k<n} k \sum_{n /(k+1)<r<n / k}\left|\mu^{2}(r)-6 / \pi^{2}\right|,
\end{aligned}
$$


since for $k>\sqrt{ } n$ the interval $(n /(k+1), n / k]$ contains at most one integer. Each integer $r \leqslant \vee n-1$ falls in exactly one such interval, and then

$$
\left|\mu^{2}(r)-6 / \pi^{2}\right| \geqslant 1-6 / \pi^{2} \text { and } k>n / r-1 .
$$

Hence, the last sum is

$$
\geqslant \frac{\left(1-6 / \pi^{2}\right)}{n} \sum_{r<\sqrt{ } n-1}(n / r-1) \rightarrow \infty \quad \text { as } n \rightarrow \infty .
$$

4. Dirichlet inverse methods. The inverse of $h(n)$ under ordinary Dirichlet convolution is denoted by $h^{*}(n)$ and is the (unique) solution of the equations

$$
\sum_{r \mid n} h(r) h^{*}(n / r)= \begin{cases}1 & (\text { if } n=1) \\ 0 & (\text { if } n \neq 1)\end{cases}
$$

In [4, Theorem 1] we gave necessary and sufficient conditions for $\left(D, h_{1}(n)\right)$ $\subset\left(D, h_{2}(n)\right)$, i.e. for every $\left(D, h_{1}(n)\right)$-summable series to be $\left(D, h_{2}(n)\right)$-summable.

THEOREM. Let

$$
\phi_{d}\left(h_{1}, h_{2}\right)=\sum_{r \mid d} h_{1}^{*}(r) h_{2}(d / r) .
$$

Then $\left(D, h_{1}(n)\right) \subset\left(D, h_{2}(n)\right)$ if and only if

$$
\sup _{m} \sum_{n=1}^{m} \frac{n}{m}\left|\Delta\left(\sum_{d<m / n} d \phi_{d}\left(h_{1}, h_{2}\right)\right)\right|<\infty,
$$

$\lim _{m \rightarrow \infty}(n / m) \Delta\left(\Sigma_{d \leqslant m / n} d \phi_{d}\left(h_{1}, h_{2}\right)\right)$ exists (each $\left.n\right)$, and

$\lim _{m \rightarrow \infty} \sum_{d=1}^{m}(d / m)[m / d] \phi_{d}\left(h_{1}, h_{2}\right)$ exists, the difference operator $\Delta$ acting on the variable $n$.

Since $\phi_{d}\left(h_{1}, h_{2}\right)=\phi_{d}\left(h_{2}^{*}, h_{1}^{*}\right)$, it follows that $\left(D, h_{1}(n)\right) \subset\left(D, h_{2}(n)\right)$ if and only if $\left(D, h_{2}^{*}(n)\right) \subset\left(D, h_{1}^{*}(n)\right)$. In particular, the corollary to [4, Theorem 1] yields

THEOREM 7. (i) $\sum a_{k}=A(D, \mu(n) / n) \Rightarrow \sum a_{k}=A \pi^{2} / 6(D, \lambda(n) / n)$.

(ii) $\sum a_{k}=A(D, \lambda(n) / n) \Rightarrow \sum a_{k}=6 A / \pi^{2}(D, \mu(n) / n)$.

Concerning equiconvergence of these last transforms we have

THEOREM 8. (i) Let $\alpha \geqslant 1$. Then with $h(n)=\lambda(n) / n$ or $\mu(n) / n$,

$$
k^{\alpha} a_{k} \rightarrow 0 \Rightarrow \sum a_{k}=0(D, h(n)) \text {. }
$$

(ii) The $(D, \mu(n) / n)$ and $\left(D, 6 \lambda(n) / \pi^{2} n\right)$ transforms are equiconvergent whenever $k^{\alpha} a_{k} \rightarrow 0$, if and only if $\alpha \geqslant 1$.

Proof. (i) Let

$$
b_{n k}=\frac{k^{1-\alpha}}{n} \sum_{r<n / k} r h(r) .
$$


It suffices to show $\left(b_{n k}\right)$ maps $c_{0}$ to $c_{0}$. First $b_{n k} \rightarrow 0$ as $n \rightarrow \infty$ (each $k$ ) since

$$
M(x)=\sum_{n<x} \mu(n)=O\left(x / \log ^{2} x\right), \quad \text { and } L(x) \equiv \sum_{n<x} \lambda(n)=O\left(x / \log ^{2} x\right)
$$

(see, e.g. Landau [5, $\S 157,167]$ ), these estimates being equivalent to the prime number theorem with an error term. Further,

$$
\begin{aligned}
\sum_{k=1}^{\infty}\left|b_{n k}\right| & \leqslant \frac{1}{n} \sum_{k \leqslant n / 2}\left|\sum_{r<n / k} r h(r)\right|+O(1) \\
& =O\left(\frac{1}{n} \sum_{k<n / 2} \frac{n}{k \log ^{2}(n / k)}\right)+O(1) \\
& =O\left(\frac{1}{n} \sum_{k<n / 2} \int_{2}^{n / k} \frac{d t}{\log ^{2} t}\right)+O(1) \\
& =O\left(\frac{1}{n} \int_{2}^{n} \frac{1}{\log ^{2} t} \sum_{k<n / t} 1 d t\right)+O(1)=O(1) .
\end{aligned}
$$

(ii) If $\alpha \geqslant 1$, equiconvergence follows trivially from (i). If $\alpha<1$,

$$
\begin{aligned}
\sum_{k=1}^{n} \mid \frac{k^{1-\alpha}}{n} & \sum_{r<n / k}\left(\mu(r)-6 \lambda(r) / \pi^{2}\right) \mid \\
& \geqslant \frac{1}{n} \sum_{n / 2<k<n} k^{1-\alpha}\left(1-6 / \pi^{2}\right) \rightarrow \infty \quad \text { as } n \rightarrow \infty .
\end{aligned}
$$

For any $\alpha$, equiconvergence whenever $k^{\alpha} a_{k} \rightarrow 0$ fails for comparisons of the $(D, \mu(n) / n)$ or $(D, \lambda(n) / n)$ transforms with any nonzero multiple of partial sums, since (2) fails. (Alternatively for $\alpha \leqslant 1$, this follows from Theorem 8(i) since $n^{\alpha} a_{n} \rightarrow 0 \neq$ convergence (to 0$)$ of $\sum a_{k}$.) Again, for comparisons of the $(D, \mu(n) / n)$ or $(D, \lambda(n) / n)$ transforms with any conservative method, equiconvergence whenever $A_{2}<\infty$ fails. This follows from Theorem 5 since

THEOREM 9. The methods $(D, \mu(n) / n),(D, \lambda(n) / n)$ are not conservative.

Proof. Necessary and sufficient conditions for $(C) \subset(D, h(n))$ were given in [4, Theorem 2], the main condition being

$$
\sup _{m} \frac{1}{m} \sum_{n=1}^{m}\left|\Delta\left(n \sum_{d<m / n} d h(d)\right)\right|<\infty .
$$

But

$$
\begin{aligned}
\frac{1}{m} \sum_{n=1}^{m}|\Delta(n M(m / n))| \geqslant \frac{1}{m} \sum_{n=1}^{m}\left|n \sum_{m /(n+1)<d<m / n} \mu(d)\right|-O(1) \\
\geqslant \frac{1}{m} \sum_{V m<n<m} \sum_{m /(n+1)<d<m / n}(m / d-1)|\mu(d)|-O(1) \\
\quad=\sum_{d<\vee m}\left|\frac{\mu(d)}{d}\right|-O(1) \geqslant \sum_{\substack{p<\vee m \\
p \text { prime }}} \frac{1}{p}-O(1) \rightarrow \infty \text { as } m \rightarrow \infty .
\end{aligned}
$$


Finally,

THEOREM 10. The $(D, \mu(n) / n)$ and $\left(D, 6 \lambda(n) / \pi^{2} n\right)$ transforms are not equiconvergent whenever $A_{2}<\infty$.

Proof.

$$
\begin{aligned}
\sum_{k=1}^{\infty} k \mid & \frac{k n^{-1} M\left(n k^{-1}\right)-6 \pi^{-2} k n^{-1} L\left(n k^{-1}\right)}{k} \\
- & \frac{(k+1) n^{-1} M\left(n(k+1)^{-1}\right)-6 \pi^{-2}(k+1) n^{-1} L\left(n(k+1)^{-1}\right)}{k+1} \mid \\
& =\left.\sum_{k=1}^{n} \frac{k}{n}\right|_{n /(k+1)<r<n / k}\left(\mu(r)-6 \lambda(r) / \pi^{2}\right) \mid \rightarrow \infty \quad \text { as } n \rightarrow \infty
\end{aligned}
$$

by the argument of Theorem 6 , whence (9) fails.

I am very grateful to the referee for his suggestions which have led to improvements in the manuscript.

\section{REFERENCES}

1. R. P. Agnew, Equiconvergence of Cesàro and Riesz transforms of series, Duke Math. J. 22 (1955), 451-460. MR 17, 146.

2. G. H. Hardy and E. M. Wright, An introduction to the theory of numbers, 4th ed., Oxford, 1960.

3. K. A. Jukes, Ordinary, strong and absolute Tauberian constants, Proc. London Math. Soc. (3) 22 (1971), 747-768. MR 45 \#773.

4. $\ldots$, On the Ingham and $(D, h(n))$ summation methods, J. London Math. Soc. (2) 3 (1971), 699-710. MR 44 \#7181.

5. E. Landau, Handbuch der Lehre von der Verteilung der Primzahlen, 2nd ed., Chelsea, New York, 1953.

6. Y. L. Luke, The special functions and their approximations, Vol. 1, Academic Press, New York, 1969.

7. I. J. Maddox, Tauberian constants, Bull. London Math. Soc. 1 (1969), 193-200. MR 40 \#596.

8. H. M. Stark, On the asymptotic density of the $k$-free integers, Proc. Amer. Math. Soc. 17 (1966), 1211-1214. MR 33 \#7310.

Department of Mathematics and Statistics, Sheffield City Polytechnic, Sheffield, ENGLAND 